> Les nanoparticules mésoporeuses ont des propriétés uniques : une grande surface spécifique ou une distribution étroite des tailles de pores. Les perspectives d'utilisation sont la création de nouveaux outils pour le diagnostic précoce. Pour ces potentielles applications biologiques, l'innocuité de ces nanoparticules doit être établie. <

\section{Nanoparticules de silice mésoporeuse optimisées pour la fluorescence à deux photons}

Valérie Lebret, Laurence Raehm, Jean-Olivier Durand, Monique Smaïhi, Corine Gerardin, Nicolas Nerambourg, Martinus H.V. Werts, Mireille Blanchard-Desce, Delphine Méthy-Gonnod, Catherine Dubernet

\section{Nanoparticules et quantum dots en imagerie cellulaire}

L'utilisation de la fluorescence dans les sciences de la vie connaît un essor important dans le domaine du diagnostic et de l'imagerie [1]. La plupart des composés développés sont des molécules organiques (fluorescéine, rhodamine) qui présentent certaines limitations dont le photoblanchiment (perte de la fluorescence). Ces limitations peuvent être levées en utilisant les nanotechnologies. Les quantum dots (nanoparticules de semi-conducteurs fluorescentes) [2] sont par exemple de plus en plus utilisées in vivo. Néanmoins, leur toxicité limite considérablement leur intérêt réel chez l'homme. L'encapsulation des fluorophores dans des nanoparticules de silice est une voie alternative explorée actuellement [3]. La matrice de silice offre l'avantage d'être inerte vis-à-vis des variations de $\mathrm{pH}$, de ne pas être sujette à des dégradations en milieu biologique et de protéger le chromophore des photodégradations. L'utilisation des nanoparticules de silice pour des applications biologiques est ainsi développée dans le cas des biodétecteurs [4], pour la reconnaissance de I'ADN [5] ou pour l'imagerie cellulaire [6]. Nous nous sommes intéressés aux nanoparticules mésoporeuses (possédant des pores de 2 à $50 \mathrm{~nm}$ ) de type MCM-4l (mobil's conception of matter). Ces nanoparticules qui ont un diamètre de l'ordre de $100 \mathrm{~nm}$ possèdent des propriétés uniques: une grande surface spécifique ou une distribution étroite des tailles de pores. Peu d'études de nanoparticules de type MCM-41 [7] fluorescentes ont été réalisées et les chromophores utilisés jusqu'à maintenant (comme la fluorescéine) sont liés de manière covalente à la matrice inorganique. Nous avons étudié l'encapsulation de nouveaux fluoropho-

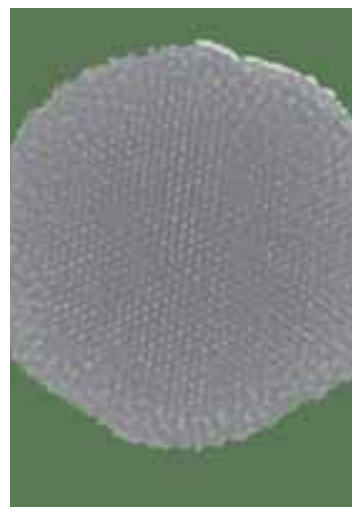

res biphotoniques (capables d'absorber simultanément deux photons) à l'intérieur des nanoparticules de type MCM-4l, une approche qui, à notre connaissance, n'a jamais été décrite [8]. L'absorption biphotonique par des fluorophores organiques connaît un intérêt croissant, en particulier dans le domaine des sciences de la vie [9]. En effet, la fluorescence biphotonique permet une résolution spatiale en trois dimensions ainsi qu'une visualisation des tissus profonds en remplaçant une excitation dans I'UVvisible par une excitation dans le proche infrarouge (moins toxique), ce qui
V. Lebret, L. Raehm, J.O. Durand, C. Gerardin : UMR 5253

CNRS-UM2-ENSCM-UMI, équipe Chimie moléculaire et organisation du solide, CC1701, Institut Charles Gerhardt, Case 1701, ICGM, Université Montpellier 2, Place Eugène Bataillon, 34095 Montpellier Cedex 05, France.

durand@univ-montp2.fr M. Smaïhi, Université Montpellier 2, Institut européen des membranes, CNRS, UMR 5635 ,

1919, route de Mende, 34293 Montpellier Cedex 05, France.

N. Nerambourg, M.H.V. Werts, M. Blanchard-Desce :

Université Rennes 1, Chimie et photonique moléculaires, CNRS, UMR 6510,

Campus de Beaulieu, 35042 Rennes Cedex, France.

D. Méthy-Gonnod,

C. Dubernet: Université Paris-Sud, Centre d'études pharmaceutiques, CNRS, UMR 8612,

5 , rue Jean-Baptiste Clément, 92296 Châtenay-Malabry Cedex, France. 


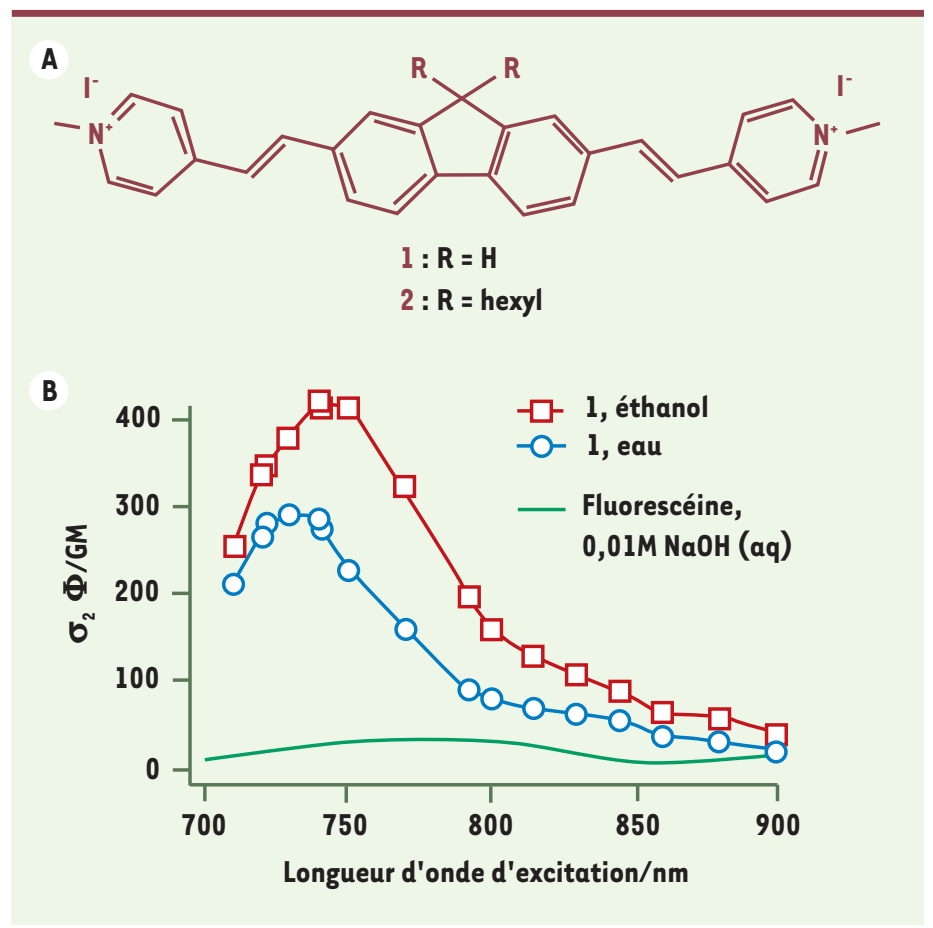

a aussi pour effet de limiter la diffusion. Une des propriétés requises pour l'utilisation de nanoparticules en imagerie cellulaire est que ces dernières possèdent une importante section efficace à deux photons (probabilité d'absorption de deux photons simultanément) dans la zone spectrale d'intérêt pour l'imagerie médicale.

\section{Pour faire une bonne nanoparticule fluorescente...}

Afin de préparer de telles nanoparticules fluorescentes, il est donc nécessaire que les fluorophores satisfassent à différents critères: solubilité dans l'eau, rendement quantique (rapport entre le nombre de photons absorbés et le nombre de photons émis) élevé et mesurable dans l'eau et surtout, une importante section efficace active en deux photons. Pour cela, nous avons synthétisé des fluorophores quadrupolaires possédant deux groupements électroattracteurs de type pyridinium $\pi$-conjugués à un cœur modulateur de type fluorényle (Figure 1).

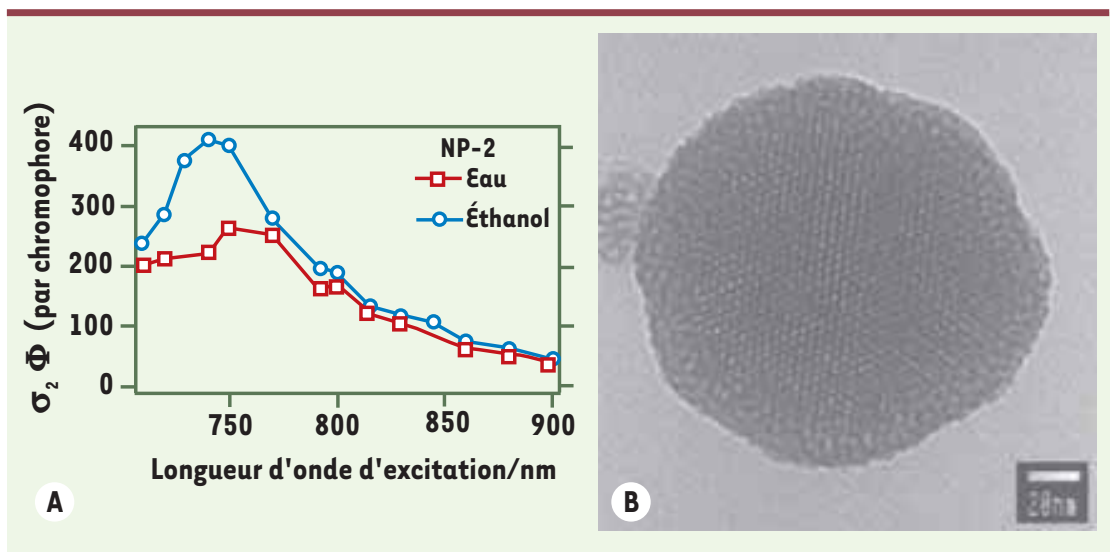

Figure 1. A. Structure des fluorophores 1 et 2. B. Spectre d'excitation biphotonique du fluorophore 1 .

Cetype de molécules est connu pour posséder d'excellentes propriétés en fluorescence biphotonique. Ces fluorophores 1 et 2 présentent des rendements quantiques respectifs dans l'eau de $35 \%$ et $27 \%$. Les propriétés d'absorption biphotonique du fluorophore 1 ont été mesurées dans l'eau. On obtient alors une excitation biphotonique dix fois plus efficace qu'avec la fluorescéine, qui est le composé de référence, utilisée dans les mêmes conditions.

Nous avons choisi d'utiliser l'inclusion stérique et les interactions électrostatiques pour immobiliser les fluorophores dans les nanoparticules. La synthèse des nanoparticules est effectuée par hydrolyse alcaline d'une solution de tétraétoxysilane (précurseur de silice) en présence des fluorophores 1 ou 2 et de bromure de cétyltriméthylammonium (tensioactif). La température et le temps de réaction sont des paramètres très importants pour contrôler la formation et la taille des nanoparticules. La microscopie électronique à transmission montre la présence de nanoparticules de diamètre $100 \mathrm{~nm}$. Ces nanoparticules possèdent des pores organisés en réseaux hexagonaux de quelques nanomètres de diamètre (visibles par microscopie électronique à transmission TEM, Figure 2B). La surface spécifique des nanoparticules est importante, de l'ordre de $700 \mathrm{~m}^{2} \mathrm{~g}^{-1}$, et le diamètre des pores est de 1,8 $\mathrm{nm}$. Les propriétés photophysiques des fluorophores ne sont pas altérées après leur encapsulation, l'absorption maximum est située à $\lambda_{\max }=420 \mathrm{~nm}$ et l'émission de fluorescence à $\lambda_{\max }=540 \mathrm{~nm}$. Grâce au spectre d'absorption des fluorophores et à la densité de la silice $(2,2 \mathrm{~kg} / \mathrm{L})$, nous avons pu déterminer le nombre de chromophores encapsulés par nanoparticule (4 400 pour 1, 8900 pour 2). Le spectre d'excitation biphotonique des nanoparticules encapsulant le fluorophore 2 (NP2) est présenté Figure 2 et la section efficace active $\left(\sigma_{2} \Phi\right)$ à deux photons est rapportée par fluorophore.

Pour les nanoparticules en solution, la détermination de la section efficace par particule conduit à une valeur géante de 10 MGM, la plus haute décrite à ce jour à notre connaissance, ce qui rend ce type de nanoparticules mésoporeuses particulièrement intéressantes pour des applications en imagerie cellulaire.

Figure 2. A. Spectre d'excitation biphotonique des NP2. B. Image TEM d'une nanoparticule. 
Parmi les perspectives d'utilisation, on peut citer la création de nouveaux outils pour le diagnostic précoce. La surface des nanoparticules pourra être fonctionnalisée par l'acide folique dans le but de cibler des cellules tumorales surexprimant les récepteurs de l'acide folique. Pour ces potentielles applications biologiques, l'innocuité de ces nanoparticules doit être établie. Dans une première approche, leur cytotoxicité a donc été testée sur des lignées de cellules cancéreuses de type MCF-7 et HeLa. Les particules encapsulant des chromophores biphotoniques présentent la même faible cytotoxicité que des nanoparticules n'ayant pas incorporé de chromophore. $\diamond$

\section{SUMMARY}

Mesoporous silica nanoparticles

for two-photon fluorescence

Mesoporous silica nanoparticles have unique properties: a specific large surface or a narrow casting of the sizes of pores. The perspectives of use are the creation of new tools for the premature diagnosis. For these potential biological applications, the harmlessness of these nanoparticles must be established. $\diamond$

\section{RÉFÉRENCES}

1. Schrock $\varepsilon$, Du Manoir S, Veldman T, et al. Multicolor spectral karyotyping of human chromosomes. Science $1996 ; 273: 494-7$.

2. Dubertret B. Boîtes quantiques en biologie : progrès récents. Med Sci (Paris) 2004 ; $20: 737-40$.

3. Santra S, Zhang P, Wang K, et al. Conjugation of biomolecules with luminophore-doped silica nanoparticles for photostable biomarkers. Anal Chem $2001 ; 73: 4988-93$.

4. Rosi NL, Mirkin CA. Nanostructures in biodiagnostics. Chem Rev 2005 ; 105 : 1547-62.

5. Lian W, Litherland Sa, Badrane H, et al. Ultrasensitive detection of biomolecules with fluorescent dye-doped nanoparticles. Anal Biochem $2004 ; 334$ : 135-44.

6. Lin YS, Tsai CP, Huang HY, et al. Well-ordered mesoporous silica nanoparticles as cell markers. Chem Mater $2005 ; 17: 4570-3$.

7. Fowler $C \varepsilon$, Khushalani D, Lebeau B, Mann S. Nanoscale materials with mesostructured interiors. Adv Mater $2001 ; 13: 649-52$.

8. Lebret V, Raehm L, Durand J0, et al. Synthesis and characterization of fluorescently doped mesoporous nanoparticles for two-photon excitation. Chem Mater $2008 ; 20: 2174-83$

9. Denk W, Strickler JH, Webb WW. Two-photon laser scanning fluorescence microscopy. Science $1990 ; 248: 73-6$.

\section{CONFLIT D'INTÉRÊTS}

Les auteurs déclarent n'avoir aucun conflit d'intérêts concernant les données publiées dans cet article.

\section{TIRÉS À PART}

J.O. Durand

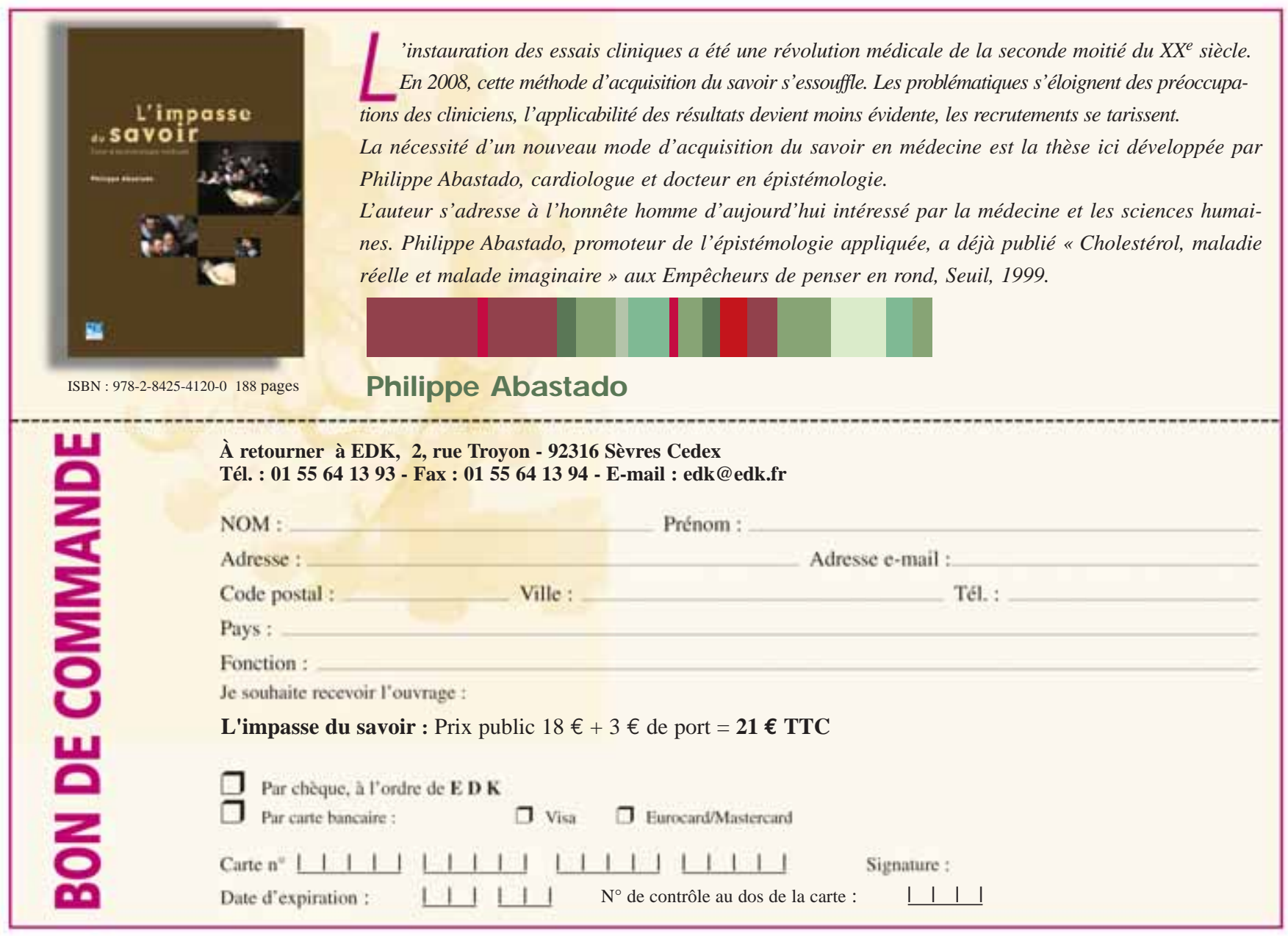

